\title{
Test Results of a Variant-Design LHC Twin-Aperture Dipole Magnet
}

\author{
C. Giloux, R. Mompo, A. Siemko, T. M. Taylor, W. Venturini Delsolaro, L. Walckiers, A. Yamamoto, T. Shintomi, \\ T. Nakamoto, N. Ohuchi, T. Ogitsu, and K. Tsuchiya
}

\begin{abstract}
Since 1989, KEK and CERN carried out jointly an experimental program in the frame of the $R \& D$ work for the $L H C$ main dipole. The mechanical structure of this design is based on a separate coil/collar and "horizontally split iron" concept. A total of four single aperture and two twin-aperture $1 \mathrm{~m}$ long dipole magnets were built. The last twin-aperture magnet was tested at CERN, reaching a maximum field of $9.55 \mathrm{~T}$ at $1.9 \mathrm{~K}$. This paper reports the magnet training performance and quench localization at $1.9 \mathrm{~K}$ and $4.5 \mathrm{~K}$. The performance as a function of current ramp rate and measurements of the field quality are also reported.
\end{abstract}

Index Terms-Magnet design, superconducting magnets.

\section{INTRODUCTION}

$\mathbf{T}$ HE DESIGN of the LHC main dipole required a long and complex collective effort. Its final product, the LHC main dipole, enters now the phase of large-scale industrial production. As in modern evolution theories, the whole process bore more resemblance to a multi-branched bush than to a straight ladder [1]: several technical solutions were considered and developed up to the stage of short models or prototypes. Some of them concerned the choice of materials or the settling of manufacturing parameters, whereas others involved deeper changes in the design philosophy. The design variant developed in collaboration by KEK and CERN since 1989 belonged to this second category. It was a small branch of the whole project, having produced in total only 4 magnets. The first two had $50 \mathrm{~mm}$ aperture, according to the original LHC specification [2]. These two magnets reached respectively $9.9 \mathrm{~T}$ and $10.3 \mathrm{~T}$ after training [3]. After the reconsideration of some key parameters of the LHC (length of the magnets, dipole field, aperture diameter), the program was redirected and targeted to a lower bore field, focusing on the ease of assembly and repeatability of the magnetic field quality in industrial production. Two $56 \mathrm{~mm}$ aperture models were built and named LHSDa01 and LHSDa02. Subsequently the LHSDa01 was slightly modified by removing the shim between collar and the iron yoke [4]. The modified LHSDa01 and the LHSDa02 reached respectively $9.77 \mathrm{~T}$ and $9.63 \mathrm{~T}$ after training. The two magnets were then assembled without a shim in a common yoke to form a twin-aperture magnet. After a 3-year long storage at room temperature, the twin-aperture magnet was

Manuscript received August 6, 2002

C. Giloux, R. Mompo, A. Siemko, T. Taylor, W. Venturini Delsolaro, and L. Walckiers are with CERN, CH-1211, Geneva 23, Switzerland (e-mail: Walter.Venturin@cern.ch).

A. Yamamoto, T. Shintomi, T. Nakamoto, N. Ohuci, T. Ogitsu, and K. Tsuchiya are with KEK, Tsukuba, Japan.

Digital Object Identifier 10.1109/TASC.2003.812658

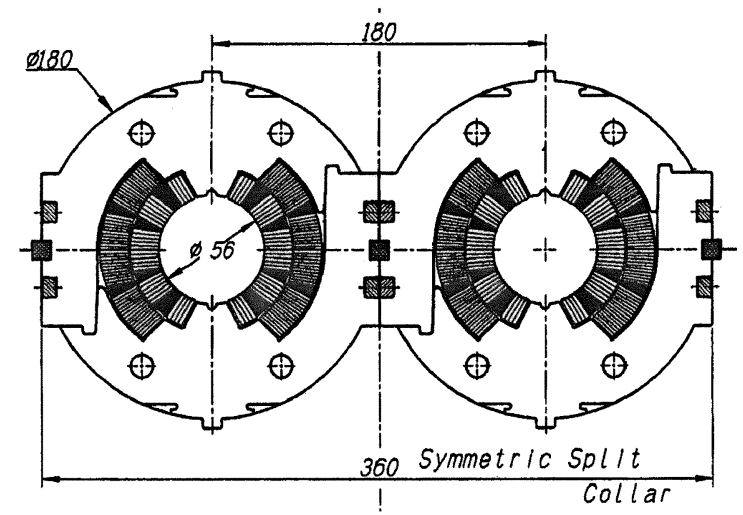

Fig. 1. Cross-section of the twin aperture dipole model.

shipped to CERN and tested in October 2001. Considering the advanced stage of the LHC project, this represented the conclusion of the program. The test results, together with a short discussion of the main design concepts, are reported here for the sake of completeness, and to document some interesting features in the quench performance.

\section{DESIGN CONCEPTS SUMMARY}

The design variants developed in the KEK/CERN collaboration were mainly mechanical. Concerning the electromagnetic design, the coil cross-section was very similar to the 2 nd generation CERN 5-blocks layout [5]. Coil ends were re-optimized, to reduce the local peak field and to enhance the mutual attraction between blocks. The cross-section of the twin aperture dipole model is shown in Fig. 1.

The principal engineering choices aimed at providing a maximum of symmetry and modularity.

\section{A. Separate Collars}

The two apertures were mechanically fully decoupled thanks to the use of separate collars. The geometry of the latter was worked out to give perfect symmetry once the two magnets are yoked together. As it is shown in Fig. 1, the yoke cavities containing the collared coils could be prolongated to form two tangent circles. The collars were designed to take $100 \%$ of the Lorenz force. No pre-stress was needed in the iron. Tapered keys are used to close the collars at the full pre-stress value at room temperature. Considerable work has been devoted to the selection of the collar material [6]. The integrated thermal shrinkage coefficients of three collar materials considered are shown in Table I, along with those of the coil and yoke. The final choice was high Mn stainless steel, KHMN, having an 
TABLE I

INTEGRAL THERMAL SHRINKAGE COEFFICIENTS

\begin{tabular}{ccccc}
\hline Coil & Iron yoke & SS SUS 316 & YUS 130 & KHMN \\
\hline $0.290 \%$ & $0.202 \%$ & $0.309 \%$ & $0.260 \%$ & $0.170 \%$ \\
\hline
\end{tabular}

integrated thermal shrinkage between $300 \mathrm{~K}$ and $1.9 \mathrm{~K}$, closest to that of the iron. Yoke and collars move together during cool down, so that the magnet section shrinks without being deformed.

\section{B. Horizontally Split Iron Yoke}

This feature was intended to provide partial support against the horizontal component of the Lorenz force. Moreover, it allowed the yoke to be used as a precise positioning tool for the two magnets. This function was achieved by means of alignment notches on top of each collar, which mated with the yoke with a $20 \mu \mathrm{m}$ tolerance. Once fixed at room temperature, the alignment is kept upon cool down. In this design, the iron could be either fully mated or there could be a gap between iron and collars, this having only a minor influence on the level of quench performance [3]. In the twin-aperture magnet, the shims between iron and collars were removed in both apertures, so there was no mechanical contribution from the yoke to the supporting of Lorenz forces. The mating of the iron yoke being a secondary issue, some tolerances on the yoke dimensions could be relaxed.

Last but not least, the horizontal split allowed easy manufacture of the yoke in two pieces.

\section{Axial Pre-Tensioning}

In dealing with the forces on the magnet ends, two approaches are currently employed; i.e., pre-compressing the heads (to compact them thus mitigating the risk of movements) or pre-tensioning them [7]. The second approach, though more difficult to implement, is an interesting option, with the pre-load acting in the same sense as the resultant Lorenz force (as it is the case for azimuthal pre-compression in the straight part). Pre-tensioning of the ends was accomplished by means of pre-tensioning bolts embedded in the collars.

\section{TEST RESUlts}

\section{A. Test Set-Up}

Tests of the twin aperture magnet were performed in a vertical cryostat at $1.9 \mathrm{~K}$ and at $4.2 \mathrm{~K}$. As the magnet had no strip heaters, the only protection was provided by an external dump resistor of $30 \mathrm{~m} \Omega$, allowing to extract about $70 \%$ of the stored energy. The coils were equipped with a set of voltage taps for quench detection and localization. Magnetic measurements were carried out by means of rotating coils [8]. The magnetic measurement shafts were also used as quench antennas, and quench localization was accomplished by combining information from the latter and from the voltage taps.

\section{B. Quench Performance}

During the first excitation at $1.9 \mathrm{~K}$ the magnet displayed a remarkable mechanical activity. Voltage spikes started to ap-

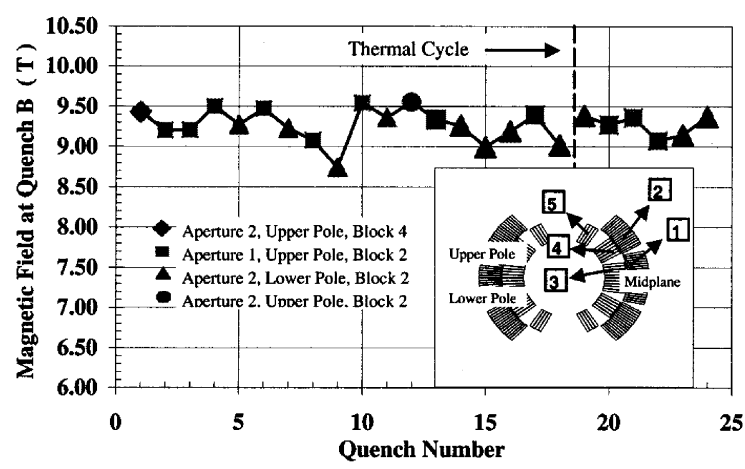

Fig. 2. Training curve at $1.9 \mathrm{~K}$. Inset: pole conventions.

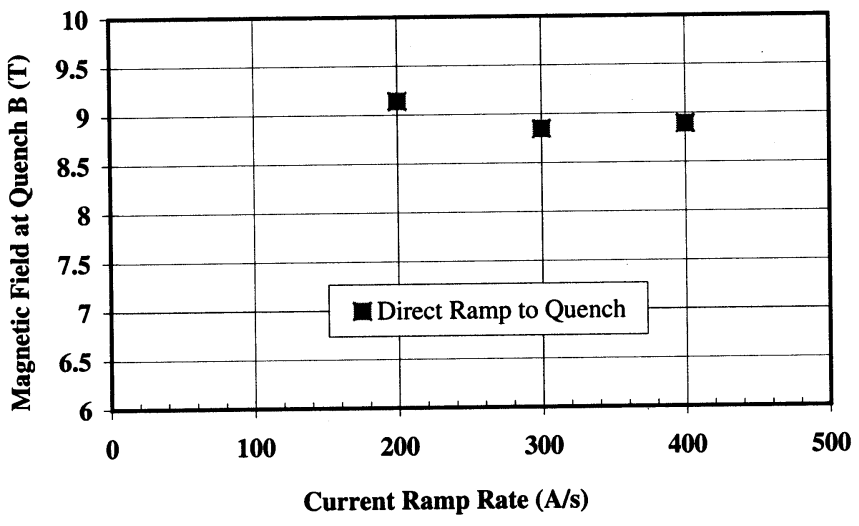

Fig. 3. Quenches at high ramp rate.

pear at less than $3 \mathrm{~T}$. The first quench, however, occurred at $9.43 \mathrm{~T}$. Subsequently, the magnet suffered from detraining and the overall quench performance was characterized by an oscillating behavior. The whole training curve is shown in Fig. 2. The maximum field of $9.55 \mathrm{~T}$ was reached at quench number 12. After the thermal cycle the magnet quenched at the same field level, though continuing to display an unstable behavior.

The quench localization analysis gave the following results: the first quench was located in aperture 2, upper pole, inner layer, block 4, while all the other quenches, with the exception of the first one, occurred in the outer layer. All quenches in aperture 1 had the same localization: upper pole, outer layer, block 2. Quenches in aperture 2 were in the two poles, but with a strong prevalence of the lower pole, outer layer (again block 2). The sequence of quench locations displays a distinct regularity: quenches in aperture 2, lower pole, block 2 (triangles in Fig. 2) tend to correspond to the de-training points, and follow, in an almost systematic way, quenches in aperture 1, upper pole, block 2 (squares in Fig. 2). Longitudinally, of a total of 24 quenches, 9 occurred in the magnet ends, 11 in the transition region and 4 in the straight part. Among the quenches in the magnet ends and in the transition region, 17 were located on the connection side (lead side) and 3 on the nonconnection side.

High ramp rate quenches were performed at $1.9 \mathrm{~K}$ after the training quenches at $10 \mathrm{~A} / \mathrm{s}$. The magnet displayed a low sensitivity to the ramp rate, as visible in Fig. 3. The measured quench current was still $11800 \mathrm{~A}$ (corresponding to $8.89 \mathrm{~T}$ ) at $400 \mathrm{~A} / \mathrm{s}$. This characteristics, already noticed [3], [4], in the single aperture models, was confirmed despite the 3 year-long period in 


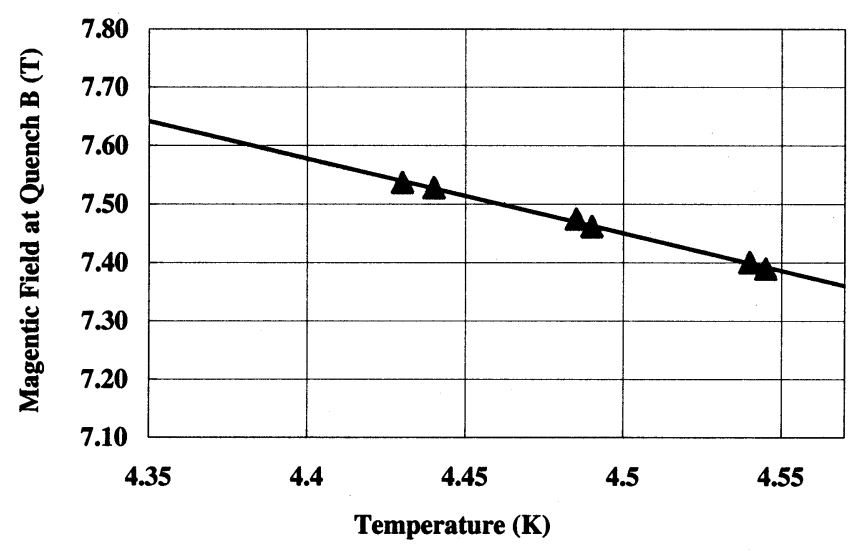

Fig. 4. Quenches around 4.5 K.

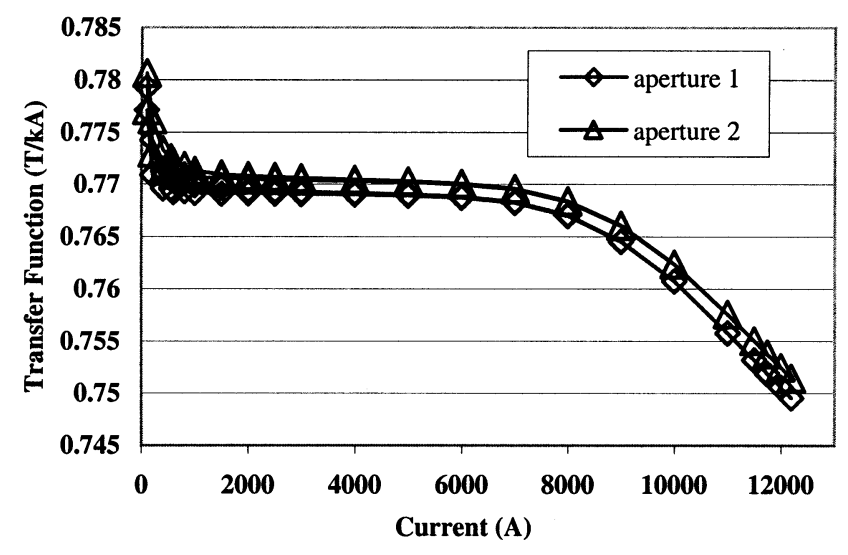

Fig. 5. Dipole transfer function.

air at room temperature, demonstrating a good stability of the inter-strand properties.

The quench performance in the vicinity of $4.5 \mathrm{~K}$ is shown in Fig. 4. All quenches at $4.5 \mathrm{~K}$ were localized in the first aperture, lower pole, inner layer, block 5, i.e., in the peak field region. This fact, as well as the good linearity and reproducibility, indicates that the points correspond, in this temperature range, to the critical surface of the wound cable.

\section{Magnetic Measurements at Cold Conditions}

An extensive characterization of the magnetic field was carried out, including measurements of the persistent current contribution to the field multipoles and of the dynamic effects (decay and snapback) [9]. The magnetic field was measured at $1.9 \mathrm{~K}$ at different currents all along the magnet load line. Multipoles averaged at $5000 \mathrm{~A}$ are hereafter called geometric. Values, in units of $10^{-4}$ of the main field, are referred to a magnetic axis defined by zeroing the 20-pole coefficient of the field expansion. The dipole transfer function and the normal quadrupole coefficient are shown in Figs. 5 and 6 respectively. The first eleven geometric multipoles are represented in Fig. 7.

Globally, the harmonic content was low. The low values of the geometric normal quadrupole terms in the two apertures were the consequence of the perfect left-right symmetry in the magnet cross-section. When compared to those of the 6-blocks design of the pre-series LHC dipoles [10], these results show a higher value of the dipole transfer function, which was also due

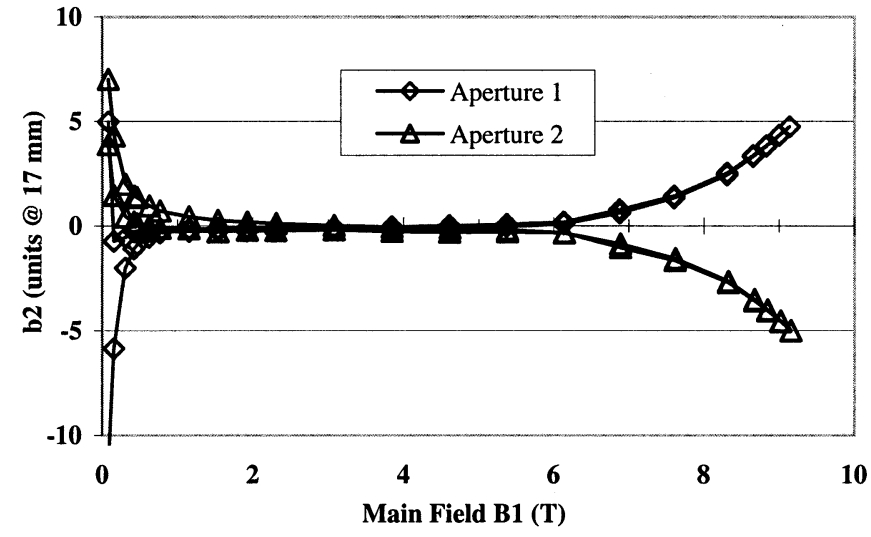

Fig. 6. Hysteresis cycle of the normal quadrupole coefficient as a function of the dipole field. Multipoles are expressed in units. One unit is $10^{-4}$ of the main field, at the reference radius of $17 \mathrm{~mm}$.

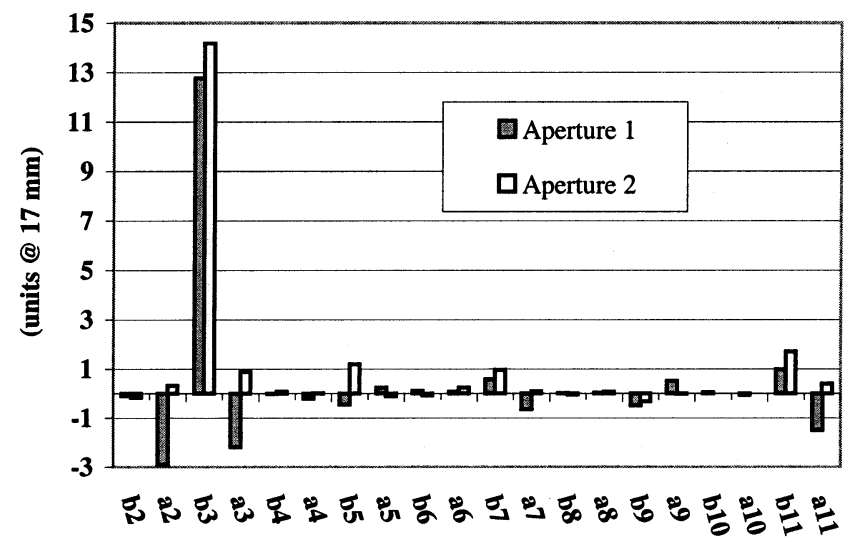

Fig. 7. Geometric multipoles: averages of the field multipole coefficients, computed at $5000 \mathrm{~A}$.

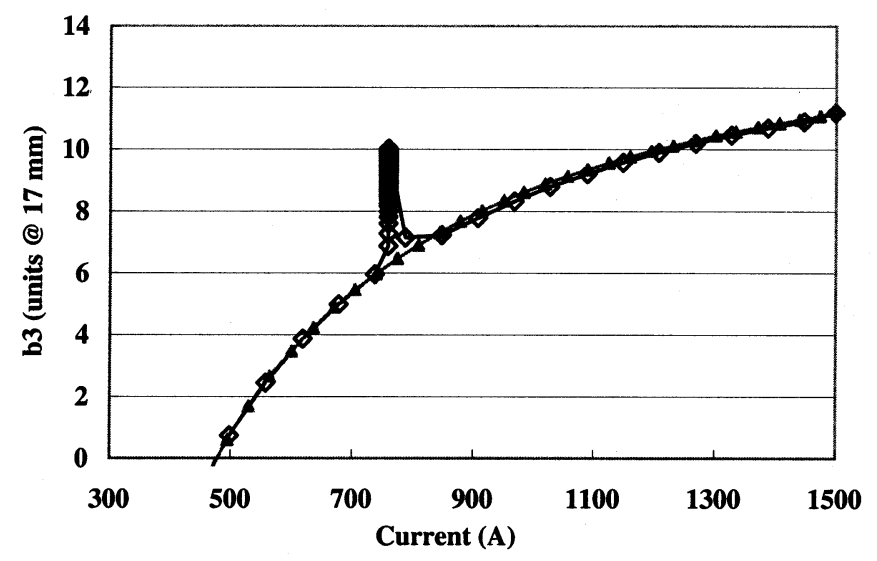

Fig. 8. Decay and snapback of the normal sextupole term during $1000 \mathrm{~s}$ at injection current (diamonds), compared to a reference measurement with a constant ramp (triangles).

to the smaller radius of the iron yoke (about $10 \%$ closer to the coils). Consequently, the effect of the iron saturation on the field harmonics was in general greater. The amplitude of the decay was also greater in the KEK magnet then in the LHC pre-series dipoles. Fig. 8 shows the decay of the normal sextupole coefficient during $1000 \mathrm{~s}$ at constant injection field. The effect of the persistent currents was comparable in the two layouts, as it depends mainly on cable properties. 


\section{DISCUSSION}

The following points can be highlighted:

1) The quench performance was only a little degraded after the yoking in the twin aperture structure and the very long storage at room temperature (3 years). In general, the equilibrium configuration of the cables at cold conditions under electromagnetic load does not coincide with that at warm conditions. However, the thermal stresses during warm up and cool down were limited by the homogeneous shrinkage coefficients of the different materials. This may explain the excellent memory displayed by the magnet. The shipping from Japan to Geneva was as well without major consequences for the quench performance.

2) All quenches except the first one were located in the outer layer. The observed modest de-training effect could be caused by differential thermal expansions due to the absence of strip heaters. As a consequence of the temperature gradient induced by the quench, the outer layer would expand more than the inner layer, thus introducing small mechanical mismatches. On the other hand, both the single aperture magnets had shown a scattered training behavior in previous tests [2]. With the connection being one in series of the two magnets, the superposition of two scattered training curves produces a scattered training curve with a lower average, as the probability to have the next point "up" is reduced to half.

3) Quenches at high ramp rate occurred close to the final training current, so that a clear identification of the type of dependence of the quench current on the ramp rate was not possible in the explored range of current ramp rates. The available data indicate that the slope is comfortably small and not of practical importance for the magnet performance.

4) This $R \& D$ program did not reach the point of trimming for the magnetic field quality. The harmonic content of the twinaperture dipole was low, with the exception of the normal sextupole, which could have easily been corrected, but the effects of the iron saturation and the decay of field harmonics were significant compared with those corresponding to the most recent design of the LHC dipole.

\section{CONCLusions}

A robust design variant for the LHC dipole resulted from the R\&D program carried out in collaboration between CERN and KEK. A small number of short dipole models were built, and a last twin-aperture model was tested at CERN in October 2001.
The magnet was excited at fields exceeding $9 \mathrm{~T}$ from the first quench. More work would be necessary to better understand the detraining phenomena, and to improve the magnetic field quality. The test results confirmed that the main engineering choices adopted in this program, i.e., the horizontally split iron, and the separate coil/collars, even unsupported by the yoke, represented valid alternative options to produce a superconducting dipole capable of satisfying the challenging specifications for the LHC accelerator.

\section{ACKNOWLEDGMENT}

The authors wish to express their gratitude to all the members of the technical teams at KEK and at CERN, which made possible the experimental program to be carried out, as well as the KEK and CERN directorates.

\section{REFERENCES}

[1] S. J. Gould, "Punctuated equilibria: An alternative to phyletic gradualism," in Models in Paleobiology, T. J. M. Schopf, Ed. San Francisco, 1972.

[2] The LHC Study Group, "Design study of the Large Hadron Collider (LHC),", CERN, CERN/91-03, May 2 1991, "Pink book".

[3] A. Yamamoto, T. Shintomi, N. Higashi, H. Hirabayashi, H. Kawamata, N. Song, A. Terashima, H. Yamaoka, S. Kawabata, G. Brianti, J. Buckley, D. Leroy, R. Perin, A. Siemko, L. Walckiers, M. Hirano, T. Origasa, K. Makishima, I. Inoue, M. Ikeda, S. Meguro, and M. Kondo, "Development of twin aperture dipole magnets for the Large Hadron Collider," IEEE Trans. App. Sup., vol. 5, no. 2, pp. 1016-1019, June 1995.

[4] T. Shintomi, T. Nakamoto, N. Higashi, N. Kimura, T. Ogitsu, K. Tanaka, A. Terashima, K. Tsuchiya, A. Yamamoto, A. Orikasa, K. Makishima, N. Siegel, D. Leroy, and R. Perin, "Test results of a single aperture dipole model magnet for the LHC," IEEE Trans. App. Sup., vol. 9, no. 2, pp. 467-470, June 1998.

[5] The LHC Study Group, "LHC, the Large Hadron Collider accelerator project," CERN/AC/-93 (LHC), November 8 1993. "White book".

[6] N. Song, A. Yamamoto, T. Shintomi, H. Yamaoka, and A. Terashima, "Field and structural analysis of $56 \mathrm{~mm}$ aperture dipole model magnets for the Large Hadron Collider," IEEE Trans. Magn., vol. 32, no. 4, pp. 2124-2127, July 1996.

[7] A. Devred, Lecture at CAS, Erice, Sicily, Italy, 2002, to be published.

[8] W. V. Delsolaro, A. Arn, L. Bottura, C. Giloux, R. Mompo, A. Siemko, and L. Walckiers, "The test facility for the short prototypes of the LHC superconducting magnets," in Proc. of the CEC/ICEC Conference, S. Breon et al., Eds: AIP, 2002, vol. 47, pp. 106-113.

[9] L. Bottura, "Field dynamics in superconducting magnets for particle accelerators," in Proceedings of the CAS Measurement and Alignment of Accelerator and Detector Magnets, S. Turner, Ed., Geneva, 1998, pp. 79-105.

[10] S. Amet, L. Bottura, V. Granata, S. Le Naour, R. K. Mishra, L. Oberli, D. Richter, S. Sanfilippo, L. Walckiers, A. Verweij, and R. Wolf, "Persistent and coupling current effects in the LHC superconducting dipoles," in this conference. 